\title{
Criminologie
}

\section{La prison de Bordeaux et sa population (de 1912 à 1940)}

\section{Dominique Delisle}

Volume 9, numéro 1-2, 1976

L’emprisonnement au Québec

URI : https://id.erudit.org/iderudit/017050ar

DOI : https://doi.org/10.7202/017050ar

Aller au sommaire du numéro

Éditeur(s)

Les Presses de l'Université de Montréal

ISSN

0316-0041 (imprimé)

1492-1367 (numérique)

Découvrir la revue

Citer cet article

Delisle, D. (1976). La prison de Bordeaux et sa population (de 1912 à 1940).

Criminologie, 9(1-2), 23-59. https://doi.org/10.7202/017050ar d'utilisation que vous pouvez consulter en ligne.

https://apropos.erudit.org/fr/usagers/politique-dutilisation/ 


\section{LA PRISON DE BORDEAUX}

ET SA POPULATION (DE 1912 à 1940)

Dominique Delisle

La prison de Bordeaux - l'actuel établissement de détention de Montréal - a été construite en vue de remplacer la vieille prison commune. En effet, elle est devenue trop petite pour recevoir les prévenus, les détenus condamnés à moins de deux ans d'emprisonnement et les sujets attendant leur transfert à l'asile, à une école de réforme pour juvéniles ou au pénitencier.

La lecture des rapports des inspecteurs des prisons de la province de Québec, d'une part, et du ministre des Travaux publics et du travail de la province de Québec, d'autre part, révèle les étapes successives ayant conduit à la fermeture, le 15 août 1913, de la prison du Pied-du-courant, sise rue Notre-Dame Est.

Le premier transfert de prisonniers, dont le nombre s'élevait à 100, de la prison du Pied-du-courant à celle de Bordeaux eut lieu le 18 novembre $1912^{1}$. La population de l'ancienne prison atteignait encore un total de 402 personnes incarcérées dont 45 attendant leur procès et 356 condamnés.

Selon le rapport de M. C.A. Vallée, premier directeur de la nouvelle prison, en date du 26 juillet $1913^{2}$. «La vieille prison du district de Montréal sera désaffectée le mois d'août prochain après le transfert du dernier contingent des prisonniers à la prison de Bordeaux....”

«En 1912, plusieurs centaines de prisonniers ont été transférés à la nouvelle prison de Bordeaux", constate-t-il. Il est donc possible

1. 43e rapport des inspecteurs des prisons de la province de Québec pour l'année 1912, p. 43, in : Documents de la session 1913, vol. 47. $n^{\circ} 4$.

2. Appendice $n^{\circ} 6$ du Rapport général du ministre des Travaux publics et du travail pour la province de Québec pour l'année finissant le 30 juin 1913, in : Documents de la session, 1913 , vol. $47, \mathrm{n}^{\circ} 3, \mathrm{p} .146$ et 147. 
de déduire de ces deux sources de renseignements que les transferts en question ont dû être effectués entre le 18 novembre 1912 et le 31 décembre de la même année.

D'autres transferts ont suivi en 1913, jusqu'à la fermeture de la prison du Pied-du-courant, le 15 août 1913. Lors d'une visite de l'inspecteur Woods à la nouvelle prison le 5 septembre 1913, il y avait 541 ( $\mathrm{sic}$ ) prisonniers dont 58 prévenus et 453 condamnés et, durant sa visite du 11 décembre 1913, 588 sujets répartis en 94 prévenus et 494 détenus 3 .

Une fois ces précisions apportées sur les premières entrées des personnes incarcérées à la prison de Bordeaux, notre analyse va porter essentiellement, sur le mouvement, ou l'évolution, de la population de cette prison depuis sa mise en fonctionnement (partielle, en 1912, complète, en 1913) jusqu'à l'année 1940 comprise. Il s'agit particulièrement d'étudier, à partir de la documentation pertinente relative à la population de l'établissement de Bordeaux, de 1912 à 1940, les données concernant les admissions en général des prisonniers et la répartition des admissions, ou des condamnations, d'après les types de délits, les types de sentences, l'âge, la profession et l'instruction.

Jusqu'à ce jour, aucune recherche de ce genre relative à la prison de Bordeaux n'a été entreprise et malgré ses limites - en raison surtout de la mauvaise qualité des statistiques de l'époque et de la valeur intrinsèquement relative du procédé de l'échantillonnage choisi - elle peut apporter une utile contribution à l'histoire des prisons du Québec.

\section{A. UTILISATION DES STATISTIQUES DES ÉTABLISSEMENTS PÉNITENTIAIRES POUR LES ANNÉES 1912-1930}

La première source de renseignements concernant la population de la prison de Bordeaux depuis son ouverture se trouve dans "les statistiques des établissements pénitentiaires (prisons, écoles de réforme et écoles d'industrie)", ainsi nommées et contenues dans les documents de la session, à la suite des rapports des inspecteurs des prisons de la province de Québec. Il s'agissait donc pour nous

3. 44e Rapport des inspecteurs des prisons de la province de Québec pour l'année 1913, p. 37, in : Documents de la session, 1914, vol. 48, $\mathrm{n}^{\circ} 4$. L'un des chiffres 541,58 et 453 doit être erroné, car le total de 58 et 453 ne correspond pas à 541 . 
de reproduire les tableaux présentés dans ces documents, puis de ne retenir et commenter que les résultats spécifiques à l'objet de la recherche, soit les types de délits, les types de sentences, l'amende, les raisons de la libération, l'âge, la récidive, la profession et l'instruction.

Plusieurs remarques sont à faire au sujet de ces variables :

a) Il est à noter qu'il n'y a des renseignements concernant l'amende pour cette période 1912-1930, que pour les années 1912 à 1915 et 1918 et 1920 ;

b) En outre, les informations concernant la récidive - lorsqu'elles existent (notamment quant au nombre des entrées et sorties annuelles des récidivistes et aux motifs de leurs condamnations) - ne sont rapportées que pour les années 1914-1924;

En conséquence de cette absence ou insuffisance de renseignements sur l'amende et la récidive, absence qui se renouvelle quant à la récidive à partir de 1935 , nous avons exclu ces deux variables de l'analyse des résultats;

c) Nous avons restreint la classification des types de délits à 17 catégories (assauts, flânerie, fauteur, ivresse, infraction aux lois municipales, fréquentation de maison de débauche, entretien de maison de débauche, vente de boisson sans licence, vagabondage, vol avec effraction, vol par serviteur, autres vols, tentative de vol, recel, dommage à la propriété, meurtre et tentative de meurtre, autres causes), telles que retenues dans les documents parlementaires (et ce, seulement à partir de 1914) afin de correspondre davantage aux classes de délits constituées pour la période suivante.

Le regroupement des nouvelies catégories comprend :

- Biens sans et avec violence : vol avec effraction, vol par serviteur, autres vols, tentative de vol, recel, dommage à la propriété, fauteur.

- Crimes contre les personnes : assauts, meurtres et tentatives de meurtres.

- Mœurs : fréquentation et entretien de maison de débauche.

- Drogues et alcoolisme : vente de boisson sans licence.

- Nuisances publiques sans victime : flânerie, vagabondage, ivresse. 
- Infractions aux lois municipales : idem (titre correspondant à son contenu).

- Autres causes : À la différence de la catégorie «autres causes" constituée pour la période 1931-40 en connaissance de son contenu, il est impossible par contre, de savoir ce que cette catégorie recouvre ici.

d) L'évolution de la population d'après le niveau d'instruction sera étudiée seulement de 1912 à 1930 et d'après la profession de 1914 à 1930, en raison de l'absence de renseignements pour les autres années.

e) Du fait que les variables qui importaient à notre étude n'ont été enregistrées dans les statistiques officielles que jusqu'en 1930 inclus, nous avons dû rechercher une autre source d'information.

\section{B. UTILISATION DES STATISTIQUES DES ÉTABLISSEMENTS PÉNITENTIAIRES POUR 1931-1940 :}

Nous avons alors eu recours pour les années suivantes (19311940) au procédé de l'échantillonnage, à partir des renseignements notés dans les registres d'écrou de la prison de Bordeaux - et parfois, à titre de vérification, dans les registres des libérations de cette même prison - dont l'accès et l'utilisation nous ont été assurés par les Archives nationales du Québec à Montréal.

\section{L'ÉCHANTILLONNAGE}

N'ayant pu, dans le temps dont nous disposions, relever toutes les informations propres à chaque prisonnier admis de 1931 à 1940 à la prison de Bordeaux, nous avons retenu un cas sur 40 . La représentativité obtenue peut être considérée comme valable, compte tenu d'un total annuel de prisonniers admis variant de 6400 à 9300 environ.

Il en ressort que l'échantillon varie entre un maximum de 233 prisonniers, en 1931, et un minimum de 161, en 1936.

\section{LA CUEILLETTE DES DONNEEES}

Les données ont été ramassées en fonction de l'objectif de notre étude. Il nous a paru intéressant de considérer l'évolution 
de la population de 1931 à 1940, sous les mêmes angles que ceux retenus pour la période précédente, c'est-à-dire, les types de délit, les types de sentence, l'amende, les raisons de la libération, l'âge, la récidive. Cependant, pour les motifs déjà indiqués, nous n'avons pas étudié le mouvement de la population d'après la récidive ou les condamnations à l'amende. De même, faute de renseignements dans les registres d'écrou concernant le niveau d'instruction et faute d'avoir relevé les informations relatives au métier ou à l'occupation, nous avons exclu de l'analyse des résultats, quant à cette période, l'instruction et la profession.

\section{LES ADMISSIONS DU 1-1-1912 AU 31-12-1940}

Dans la première partie de cette étude, il est intéressant d'analyser tout d'abord l'évolution de la population générale admise à l'établissement de détention de Montréal de 1912 à 1940, telle que nous l'indiquons ci-dessus:

TABLEAU 1

Nombre d'admissions de 1912 à 1940

\begin{tabular}{lllllr}
\hline Année & $\mathrm{Nb}$ & Année & $\mathrm{Nb}$ & Année & $\mathrm{Nb}$ \\
\hline 1912 & 5679 & 1922 & 5510 & 1932 & 7920 \\
1913 & 7193 & 1923 & 5234 & 1933 & 7278 \\
1914 & 6677 & 1924 & 6026 & 1934 & 7174 \\
1915 & $5071^{1+1}$ & 1925 & 6612 & 1935 & 7164 \\
1916 & 3398 & 1926 & 5677 & 1936 & 6422 \\
1917 & 3615 & 1927 & 6373 & 1937 & 7444 \\
1918 & 3956 & 1928 & 6089 & 1938 & 7631 \\
1919 & 4321 & 1929 & 7258 & 1939 & 6949 \\
1920 & 4407 & 1930 & 6990 & 1940 & 6603 \\
1921 & 6618 & 1931 & 9284 & & \\
\hline
\end{tabular}

L'examen du tableau 1, appelle différentes constatations :

Bien qu'aucune remarque n'ait été faite à ce sujet sous les tableaux se rapportant aux années 1912 et 1913, il faut supposer que les admissions qui s'élèvent à 5679 , en 1912 , et à 7193 , en 1913, concernent le total des prisonniers entrés, aussi bien, à l'ancienne prison du Pied-du-courant, fermée le 15 août 1913, qu'à la nouvelle prison de Bordeaux.

En ce qui a trait aux admissions, elles ont varié entre deux extrêmes : le plus bas taux, -3398 - en 1916, le plus haut 9284 - en 1931. Le montant inférieur des admissions en 1916

4. Les documents parlementaires révèlent dans d'autres tableaux, pour l'année 1915, un total d'admissions s'élevant à 4516 au lieu de 5071 . 
a été atteint à la suite d'une baisse marquée des entrées à la prison, en passant de 5071, ou 4516 , en 1915 à 3398 , en 1916. Ce n'est qu'en 1920 - la première guerre mondiale étant terminée - que le nombre des admissions 4407 retrouve, après une progression, le niveau qu'il avait à peu près en 1915 , soit 4516 admissions. Quant au "record" des admissions en 1931, il s'est produit de façon spectaculaire, durant cette période dite de la grande dépression économique, puisque le nombre des admissions est passé de 6990 , en 1930 à 9284 , en 1931, - augmentation de près de 2300 en un an.

Deux paliers apparaissent parallèlement :

- En 1914, 1921, 1925, 1927, 1936, et 1940, le total des admissions annuelles s'équilibre au niveau de 6400 et 6700 environ.

- Le deuxième niveau, plus élevé, se stabilise autour de 7000 à 7400 admissions par an. Il s'agit des années 1913-1929-19301933-1934-1935-1937 et 1939.

Une étude plus poussée de cette population permet de distinguer les deux catégories majeures qu'elle comprend soit les prévenus et les détenus et de suivre leur évolution respective.

Cependant les statistiques officielles relatives à la période 1912-1930 ne rapportent que le nombre de détenus et de prévenus présents le premier jour de chaque année, tel que nous le montre le tableau 2.

Celui-ci révèle ainsi que le pourcentage de détenus s'élève le plus souvent au-dessus de $80 \%$, et même en 1923 , jusqu'à $90 \%$. C'est en 1921 qu'il atteint son niveau le plus bas, soit $64,52 \%$.

Néanmoins, ces résultats sont à nuancer par l'analyse du tableau qui indique un taux moins élevé de condamnés par rapport au nombre des prisonniers entrés et sortis dans l'année.

Il est possible de conclure que l'analyse du tableau 3 , à la différence de celle du tableau 2 , révèle un pourcentage de détenus qui ne dépasse qu'une seule fois - en 1922 - les $80 \%$. Le plus souvent, il s'échelonne entre $60 \%$ et $70 \%$. Cette différence s'explique par le fait que les détenus séjournent plus longtemps en institution.

Quant à la période suivante (1931-1940), un calcul approximatif du nombre de détenus et de prévenus peut être fait à partir des résultats donnés par l'ordinateur relativement au pourcentage 
TABLEAU 2

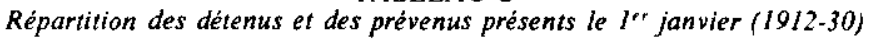

\begin{tabular}{|c|c|c|c|c|c|c|}
\hline \multirow{2}{*}{ Année } & \multicolumn{2}{|c|}{ Détenus } & \multicolumn{2}{|c|}{ Prévenus } & \multicolumn{2}{|c|}{ Population totale } \\
\hline & $\mathrm{Nb}$ & $\%$ & $\mathrm{Nb}$ & $\%$ & $\mathrm{Nb}$ & $\%$ \\
\hline 1912 & 368 & 87,82 & 51 & 12,17 & 419 & 99,99 \\
\hline 1913 & 334 & 87,66 & 47 & 12,33 & 381 & 99,99 \\
\hline 1914 & 502 & 84,09 & 95 & 15,91 & 597 & 100,00 \\
\hline 1915 & 490 & 88,29 & 65 & 11,71 & 555 & 100,00 \\
\hline 1916 & 270 & 87,67 & 38 & 12,33 & 308 & 100,00 \\
\hline 1917 & 234 & 84,48 & 43 & 15,52 & 277 & 100,00 \\
\hline 1918 & 234 & 78,26 & 65 & 21,74 & 299 & 100,00 \\
\hline 1919 & 254 & 85,81 & 42 & 14,19 & 296 & 100,00 \\
\hline 1920 & 204 & 71,33 & 82 & 28,67 & 286 & 100,00 \\
\hline 1921 & 211 & 64,52 & 116 & 35,48 & 327 & 100,00 \\
\hline 1922 & 329 & 81,03 & 77 & 18,97 & 406 & 100,00 \\
\hline 1923 & 459 & 90,00 & 51 & 10,00 & 510 & 100,00 \\
\hline 1924 & 315 & 82,46 & 67 & 17,54 & 382 & 100,00 \\
\hline 1925 & 373 & 80,74 & 89 & 19,26 & 462 & 100,00 \\
\hline 1926 & 413 & 84,11 & 78 & 15,89 & 491 & 100,00 \\
\hline 1927 & 343 & 80,52 & 83 & 19,48 & 426 & 100,00 \\
\hline 1928 & 332 & 74.94 & 111 & 25.06 & 443 & 100,00 \\
\hline 1929 & 388 & 81,00 & 91 & 19,00 & 479 & 100,00 \\
\hline 1930 & 397 & 73,65 & 142 & 26,34 & 539 & 99,99 \\
\hline
\end{tabular}

TABLEAU 3

Pourcentage de détenus par rapport aux admissions et aux sorties

\begin{tabular}{|c|c|c|c|c|c|}
\hline \multirow{3}{*}{ Année } & \multirow{3}{*}{$\frac{\text { Admissions }}{\mathrm{Nb}}$} & \multirow{3}{*}{$\frac{\text { Sorties }}{\mathrm{Nb}}$} & \multirow{3}{*}{$\mathrm{Nb}$} & \multicolumn{2}{|c|}{ Détenus } \\
\hline & & & & $\%$ par $\mathrm{r}$ & it aux \\
\hline & & & & Admissions & Sorties \\
\hline 1912 & 5679 & 5717 & 3890 & 68,49 & 68.04 \\
\hline 1913 & 7193 & 6977 & 5540 & 77,01 & 79,40 \\
\hline 1914 & 6677 & 6719 & 4916 & 73,63 & 73,17 \\
\hline 1915 & 5071 & 5318 & 3203 & 63,16 & 60,23 \\
\hline 1916 & 3398 & 3429 & 2231 & 65,66 & 65,06 \\
\hline 1917 & 3615 & 3593 & 2201 & 60,88 & 61,26 \\
\hline 1918 & 3956 & 3959 & 2546 & 64,36 & 64,31 \\
\hline 1919 & 4321 & 4331 & 3004 & 69,52 & 69,36 \\
\hline 1920 & 4407 & 4366 & 3004 & 68,16 & 68,80 \\
\hline 1921 & 6618 & 6539 & 4920 & 74,34 & 75,24 \\
\hline 1922 & 5510 & 5406 & 4409 & 80,02 & 81,56 \\
\hline 1923 & 5234 & 5362 & 3676 & 70,23 & 68,56 \\
\hline 1924 & 6026 & 5946 & 4081 & 67,72 & 68,63 \\
\hline 1925 & 6612 & 6583 & 4651 & 70,34 & 70,65 \\
\hline 1926 & 5677 & 5742 & 3724 & 65,60 & 64,85 \\
\hline 1927 & 6373 & 6356 & 4225 & 66,29 & 66,47 \\
\hline 1928 & 6089 & 6053 & 3911 & 64,23 & $64,6]$ \\
\hline 1929 & 7258 & 7198 & 4724 & 65,09 & 65,63 \\
\hline 1930 & 6990 & 6849 & 4679 & 66,94 & 68,32 \\
\hline
\end{tabular}


TABLEAU 4

Pourcentage d'incarcérés d'après les raisons de leur libération

\begin{tabular}{|c|c|c|c|c|c|c|c|c|c|c|c|c|}
\hline Année ${ }^{\mathrm{A}}$ & $\begin{array}{l}\text { Plainte retirée } \\
\text { Acquittement } \\
\text { Sine die } \\
\text { (1) }\end{array}$ & $\begin{array}{l}\text { Fin } \\
\text { de } \\
\text { sentence } \\
(2)\end{array}$ & $\begin{array}{c}\text { Sous } \\
\text { caution } \\
(3)\end{array}$ & $\begin{array}{l}\text { Libération } \\
\text { conditionnelle } \\
\text { (4) }\end{array}$ & $\begin{array}{l}\text { Sentence } \\
\text { suspendue } \\
\text { (5) }\end{array}$ & $\begin{array}{l}\text { Parole } \\
\text { (6) }\end{array}$ & $\begin{array}{c}\text { Temps } \\
\text { en } \\
\text { prison } \\
(7)\end{array}$ & $\underset{\substack{\text { Amende } \\
\text { payée (cous } \\
(8)}}{ }$ & $\begin{array}{l}\text { Ordres } \\
\text { spéciaux } \\
\text { ur sup. etc. } \\
(9)\end{array}$ & $\begin{array}{l}\text { Transfert } \\
\text { au } \\
\text { pénitencier } \\
\text { (10) }\end{array}$ & $\begin{array}{l}\text { Renvoi } \\
\text { (1I) }\end{array}$ & $\begin{array}{c}\text { Autres } \\
(12)\end{array}$ \\
\hline $\begin{array}{l}1931 \\
1932 \\
1933 \\
1934 \\
1935 \\
1936 \\
1937 \\
1938 \\
1939 \\
1940\end{array}$ & $\begin{array}{r}2.6 \\
2.5 \\
3.3 \\
2.8 \\
1.7 \\
1.2 \\
.5 \\
1.6 \\
- \\
1.8\end{array}$ & $\begin{array}{l}50.2 \\
51.5 \\
44.6 \\
41.7 \\
43.3 \\
57.8 \\
58.3 \\
51.6 \\
45.7 \\
56.6\end{array}$ & $\begin{array}{r}13.3 \\
10.6 \\
13.0 \\
15.6 \\
20.0 \\
20.5 \\
16.6 \\
8.9 \\
16.8 \\
8.4\end{array}$ & $\begin{array}{r}2.1 \\
2.5 \\
3,3 \\
1,7 \\
.6 \\
.5 \\
1.6 \\
1.7 \\
-\end{array}$ & $\begin{array}{r}3.0 \\
4.0 \\
2.7 \\
1.7 \\
.6 \\
4.3 \\
3.2 \\
2.1 \\
2.3 \\
1.8\end{array}$ & $\begin{array}{l}4,3 \\
5,1 \\
5,4 \\
6,1 \\
3,9 \\
1,9 \\
2,1 \\
5,7 \\
5,8 \\
6,6\end{array}$ & $\begin{array}{l}3,4 \\
1,5 \\
3,8 \\
3,9 \\
6,1 \\
1,9 \\
2,7 \\
3,6 \\
6,4 \\
3,0\end{array}$ & $\begin{array}{l}4,7 \\
6,1 \\
2,7 \\
6,1 \\
7,2 \\
4,3 \\
4,3 \\
7,3 \\
6,4 \\
4,8\end{array}$ & $\begin{array}{l}3,0 \\
2,0 \\
2,2 \\
5,0 \\
6,1 \\
2,5 \\
1,6 \\
4,2 \\
8,1 \\
3,6\end{array}$ & $\begin{array}{l}5.6 \\
7.1 \\
8,7 \\
7.8 \\
2.8 \\
1,2 \\
5,3 \\
6.8 \\
1,2 \\
8,4\end{array}$ & $\begin{array}{r}4,3 \\
3,5 \\
2,7 \\
1,7 \\
2,2 \\
1,2 \\
1,1 \\
2,1 \\
1,7 \\
.6\end{array}$ & $\begin{array}{l}3.4 \\
3.5 \\
7,6 \\
6,1 \\
6,1 \\
2.5 \\
3.7 \\
4,7 \\
4,0 \\
4.2\end{array}$ \\
\hline $\begin{array}{l}\text { Moyenne } \\
1931-40\end{array}$ & 1.8 & 50.1 & 14.2 & 1,5 & 2,6 & 4,7 & 3.6 & 5,4 & 3.8 & 5,6 & 2,2 & 4,6 \\
\hline
\end{tabular}


d'incarcérés d'après les raisons de leur libération et contenus dans le tableau 4.

Ce tableau 4 permet de procéder à des regroupements contenus dans le tableau 5. D'un côté, l'ensemble des colonnes intitulées "fins de sentence, libération conditionnelle, amende payée, ordres spéciaux, transfert au pénitencier" ne concerne que des personnes restées en prison à titre de détenus. De l'autre, l'addition des colonnes aplainte retirée - acquittement - sine die, sous caution, sentence suspendue, parole, temps en prison, renvoin nous donne le total des prévenus. Enfin, la colonne "autres» comprend aussi bien des détenus que des prévenus.

Il résulte du tableau 5 que le pourcentage de détenus, de 1931 à 1940 est comparable aux résultats rapportés dans le tableau 3 , puisqu'il varie en général entre $60 \%$ et $75 \%$.

TABLEAU 5

Répartition des prévenus et des détenus admis de 1931 a 1940 ,

calculée à partir du tableau 4

\begin{tabular}{|c|c|c|c|c|}
\hline Année & Prévenus & Détenus & $\begin{array}{c}\text { Autres } \\
\text { (détenus-prévenus) }\end{array}$ & Total \\
\hline 1931 & 30.9 & 65.6 & 3.4 & 99.9 \\
\hline 1932 & 27,2 & 69.2 & 3.5 & 99.9 \\
\hline 1933 & 30,9 & 61.5 & 7,6 & 100.0 \\
\hline 1934 & 31,8 & 62,3 & 6.1 & 100,2 \\
\hline 1935 & 34,5 & 59,4 & 6.1 & 100.0 \\
\hline 1936 & 31,0 & 66,4 & 2.5 & 99,9 \\
\hline 1937 & 26,2 & 70.0 & 3.7 & 99.9 \\
\hline 1938 & 24.0 & 71,5 & 4.7 & 100.2 \\
\hline 1939 & 33,0 & 63,1 & 4,0 & 100,1 \\
\hline 1940 & 22,2 & 73.4 & 4,2 & 99.8 \\
\hline \multicolumn{2}{|c|}{ Moyenne $1931-194029,1$} & 66,4 & 4,6 & 100,1 \\
\hline
\end{tabular}

\section{LA RÉPARTITION DES ADMISSIONS OU DES CONDAMNATIONS SELON LE TYPE DE DÉLIT}

Étant donné que, d'une part, les résultats concernant les types de délit sont considérés dans les documents de la Session en regard seulement des détenus, alors que, de 1931 à 1940, ils le sont d'après la grandeur totale des échantillons et, d'autre part, le contenu des catégories de délits n'est pas tout à fait pareil selon qu'il s'agisse de l'une ou l'autre période, c'est en deux temps qu'a été faite l'étude 
TABLEAU 6

Répartition des détenus selon le type de délits

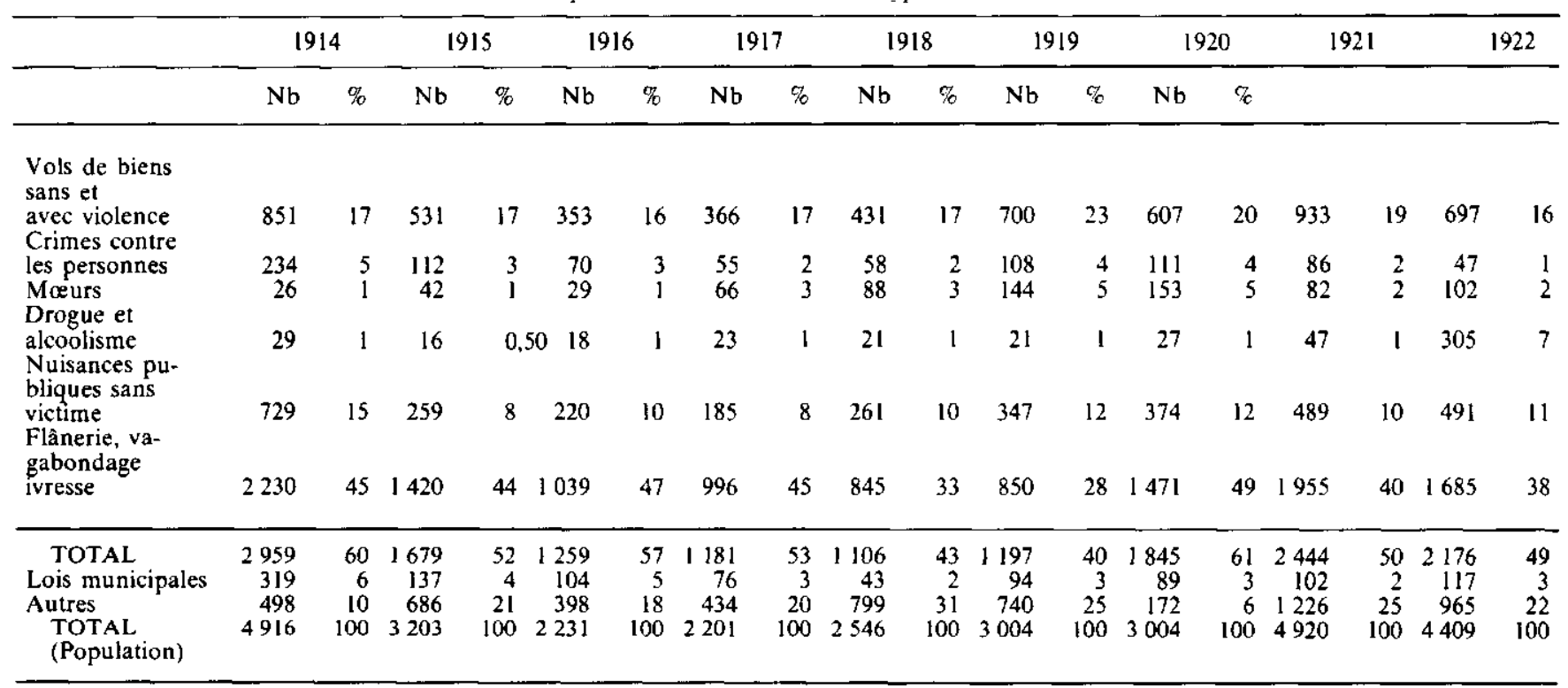


TABLEAU 6A (SUITE)

Répartition des détenus selon le type de délits

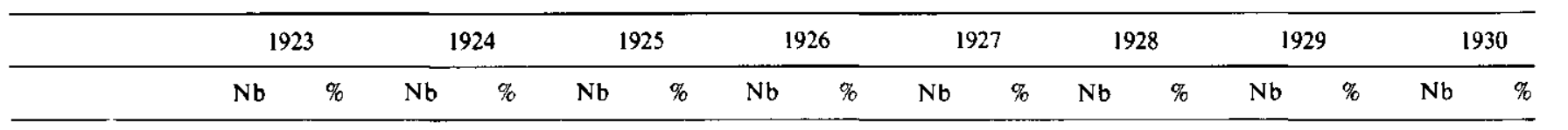

Vols de biens

sans et

avec violence

Crimes contre

les personnes

Mours

Drogue et

alcoolisme

Nuisances pu-

bliques sans

victimes

Flânerie, va-

gabondage

$523 \quad 14 \quad 714$

$17 \quad 966$

$21 \quad 846$

$\begin{array}{rrrrr}55 & 1 & 69 & 2 & 131 \\ 133 & 4 & 112 & 3 & 123\end{array}$

$435 \quad 12$

485

$123 \quad 3$

$268 \quad 7 \quad 352$

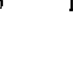

ivresse

$1390 \quad 38 \quad 1408$

TOTAL

Lois municipales

Autres

$1658 \quad 45 \quad 1760$

TOTAL

$\begin{array}{rrrrr}35 & 45 & 1760 & 43 & 2098\end{array}$

(Population)

$\begin{array}{lll}837 & 23 & 924\end{array}$

$0.42 \quad 80$

$\begin{array}{lrrr}0.42 & 80 & 2 & 39 \\ 23 & 645 & 14 & 757\end{array}$

3676

$\begin{array}{llll}14 & 757 & 20 & 589\end{array}$

521

$\begin{array}{lll}846 & 23 & 71 \\ 34 & 1 & 7 \\ 82 & 2 & 75\end{array}$

8

631 17

11

$17 \quad 822$

21

949

$20 \quad 1261$

27

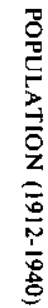


du mouvement de la population de la prison en considération de la cause de la condamnation.

Répartition des détenus selon le type de délit (1914-1930)

D'après les tableaux 6-6A, l'ordre décroissant d'importance selon lequel les infractions ont été commises est le suivant :

Les nuisances publiques sans victime - spécialement l'ivresse - :

Le pourcentage de cas d'ivresse varie de près du tiers à la moitié, notamment, en 1920 , il atteint $49 \%$. Il y a une exception, en 1930 , où l'on trouve un minimum de $18 \%$ de sujets condamnés pour ivresse. Cette année-là, le pourcentage le plus élevé de délits concernait les vols des biens sans et avec violence $27 \%$.

Cependant, dans tous les cas, c'est la catégorie des nuisances publiques sans victime qui vient constamment en premier lieu, en variant de $31 \%$, en 1930 à $61 \%$, en 1920 .

En seconde position se trouve la classe des vols de biens sans et avec violence (minimum : 14\%, en $1923-$ maximum $=23 \%$ en 1919).

Au même niveau, le groupe des infractions intitulé "Autres" (dont les documents officiels ne donnent aucune description) comporte une variation de $6 \%$ en 1920 à $31 \%$ en 1918 , assez comparable à celles des vols de biens sans et avec violence. Selon les années, l'importance numérique de ce groupe, dépasse ou suit cette catégorie.

Les accusés de flâneries et de vagabondages forment aussi un groupe assez substantiel variant de $7 \%$ en 1923 , à $16 \%$ en 1929 .

La catégorie "drogue et alcoolisme», qui ne se rapporte, pour cette période qu'aux ventes de boissons «sans licence», alors qu'elle n'avait impliqué qu'un nombre minime de prisonniers pendant huit ans s'est subitement gonflée, en passant de $0,95 \%$ en 1921 à $7 \%$ en 1922. À partir de 1922, son taux dépasse toujours celui des crimes contre les personnes, puisqu'il varie de $8 \%$ en 1930 à $17 \%$ en 1926.

Les infractions aux lois municipales, de même que les deux autres types de délits, (mœurs et crimes contre les personnes), atteignent un taux inférieur à $10 \%$. On peut donc placer ces trois catégories en queue de liste. Selon les années, l'une prend le pas 
sur l'autre, mais de toute façon, leur degré d'importance vient toujours après les cinq classes précédemment citées.

Répartition des échantillons selon le type de délits (1931-40)

Les tableaux 7-7A indiquent que les types de délits se classent entre 1931-1940 dans un ordre d'importance différent de celui de la période précédente.

Cette fois-ci, le groupe des délits contre les biens, sans et avec violence, se situe à la première place. Les pourcentages varient entre un minimum de $38 \%$ en 1940 et un maximum de $55 \%$ en 1936. Ils sont donc plus élevés que ceux qui s'échelonnaient entre les extrêmes $14 \%$ et $23 \%$ au cours de la période 1914-1930.

La catégorie des délits de «nuisances publiques sans victime», cédant sa préséance des années 1914-1930, prend ici le deuxième rang. Les pourcentages la concernant sont de beaucoup inférieurs aux niveaux qu'ils atteignaient auparavant. Ils varient en effet de $14 \%$ en 1933 à $26 \%$ en 1940 .

Les délits relatifs aux drogues et à l'alcoolisme viennent en troisième position, au moins pour les années 1931, 1932, 1934, 1935, 1939 et 1940 , en variant de $9 \%$ en 1940 à $15 \%$ en 1934 . Quant aux autres années, le taux est inférieur avec un minimum de $4 \%$ en 1936 et 1937 et un maximum de $8 \%$ en 1933.

Le total des catégories $7,9,10,11$, soit celles relatives «aux nuisances publiques avec victimes», «infractions contre l'État», «auto» et autres permet d'obtenir une catégorie numériquement comparable à celle intitulée "autres» pour la période 1914-1930. $\mathrm{Ce}$ nouvel ensemble se situe alors en pourcentage au même niveau à peu près que les délits udrogue et alcoolisme», avec un minimum de $5 \%$ en 1936 et un maximum de $15 \%$ en 1935 .

Les infractions contre les mours forment une catégorie plus vaste que celle qui ne concernait, de 1914 à 1930, que la fréquentation et l'entretien des maisons de débauche. Aussi ne faut-il pas s'étonner d'un niveau supérieur des taux qui varient de $3 \%$ en 1935 à $9 \%$ en 1931 .

Les crimes contre les personnes suivent de près la précédente catégorie et même - certaines années - ils la dépassent en pourcentage, notamment en 1932 (7\% contre 6\%), en 1935 (6\% contre $3 \%$ ), en 1939 ( $9 \%$ contre $7 \%$ ). Ils leur sont égaux deux fois, en 1933 , avec $9 \%$, et en 1934 , avec $6 \%$. 
TABLEAU 7

Répartition du pourcentage des détenus selon

le type de délit

\begin{tabular}{|c|c|c|c|c|c|c|}
\hline & 1931 & 1932 & 1933 & 1934 & 1935 & 1936 \\
\hline & $\%$ & $\%$ & $\%$ & $\%$ & $\%$ & $\%$ \\
\hline $\begin{array}{l}\text { 1. Vols sans violence } \\
\text { de biens } \\
\text { 2. avec violence } \\
\text { TOTAL }\end{array}$ & $\begin{array}{r}37 \\
3 \\
40\end{array}$ & $\begin{array}{r}37 \\
3 \\
40\end{array}$ & $\begin{array}{r}42 \\
5 \\
47\end{array}$ & $\begin{array}{r}40 \\
4 \\
44\end{array}$ & $\begin{array}{r}44 \\
3 \\
47\end{array}$ & $\begin{array}{r}52 \\
3 \\
55\end{array}$ \\
\hline $\begin{array}{l}\text { 3. Crimes contre } \\
\text { les personnes } \\
\text { 4. Mours } \\
\text { 5.Drogue et }\end{array}$ & $\begin{array}{l}7 \\
9\end{array}$ & $\begin{array}{l}7 \\
6\end{array}$ & $\begin{array}{l}9 \\
9\end{array}$ & $\begin{array}{l}6 \\
6\end{array}$ & $\begin{array}{l}6 \\
3\end{array}$ & $\begin{array}{l}5 \\
7\end{array}$ \\
\hline $\begin{array}{l}\text { 6. Nuisances sans victime } \\
\text { 7. publiques avec victime } \\
\text { 8. Lois municipales et }\end{array}$ & $\begin{array}{r}15 \\
17 \\
3\end{array}$ & $\begin{array}{r}14 \\
19 \\
2\end{array}$ & $\begin{array}{r}8 \\
15 \\
3\end{array}$ & $\begin{array}{r}15 \\
16 \\
2\end{array}$ & $\begin{array}{r}10 \\
17 \\
2\end{array}$ & $\begin{array}{r}4 \\
19 \\
1\end{array}$ \\
\hline $\begin{array}{l}\text { particulières } \\
\text { 9. Infractions contre }\end{array}$ & I & 2 & 3 & 1 & 3 & 4 \\
\hline $\begin{array}{l}\text { l'État } \\
\text { 10. Auto } \\
\text { 11. Autres }\end{array}$ & $\overline{3}$ & $\begin{array}{l}- \\
4 \\
5\end{array}$ & $\begin{array}{l}1 \\
4 \\
3\end{array}$ & $\begin{array}{l}1 \\
4 \\
5\end{array}$ & $\begin{array}{l}2 \\
6 \\
4\end{array}$ & $\begin{array}{l}1 \\
4 \\
1\end{array}$ \\
\hline $\begin{array}{l}\text { TOTAL } \\
\text { (échantillon) }\end{array}$ & 100 & 100 & 100 & 100 & 100 & 100 \\
\hline Autres (total des catégories $7,9,10,11$ ) & 12 & 11 & 10,5 & 12 & 14 & 7 \\
\hline
\end{tabular}


TABLEAU 7A (SUITE)

Répartition des prisonniers selon le type de délits

\begin{tabular}{|c|c|c|c|c|c|}
\hline & 1937 & 1938 & 1939 & 1940 & 1931 a 1940 \\
\hline & $\%$ & $\%$ & $\%$ & $\%$ & $\%$ \\
\hline \multicolumn{6}{|l|}{ 1. Vols sans violence } \\
\hline de biens & 37 & 46 & 38 & 33 & 40 \\
\hline 2. avec violence & 4 & 2 & 1 & 5 & 3 \\
\hline & 41 & 48 & 39 & 38 & 43 \\
\hline \multicolumn{6}{|l|}{ 3. Crimes contre } \\
\hline les personnes & 5 & 5 & 9 & 2 & 6 \\
\hline 4. Mœurs & 10 & 8 & 7 & $\overline{7}$ & 7 \\
\hline \multicolumn{6}{|l|}{ 5. Drogue et } \\
\hline alcoolisme & 4 & 5 & 14 & 9 & 10 \\
\hline 6. Nuisances sans victime & 25 & 25 & 20 & 26 & 20 \\
\hline 7. publiques avec victime & 2 & 1 & 2 & 3 & 2 \\
\hline \multicolumn{6}{|l|}{ 8. Lois municipales et } \\
\hline \multicolumn{6}{|l|}{ 9. Infractions contre } \\
\hline l'État & 1 & 0,5 & - & 4 & 1 \\
\hline 10. Auto & 3 & 3 & 2 & i & 3 \\
\hline 11. Autres & 3 & 5 & 3 & 4 & 4 \\
\hline $\begin{array}{l}\text { TOTAL } \\
\text { (échantillon) }\end{array}$ & 100 & 100 & 100 & 100 & 100 \\
\hline Autres (Total des catégories $7,9,10,11$ ) & 9 & 8.5 & 8 & 12 & 10 \\
\hline
\end{tabular}


Les infractions aux lois municipales et particulières se situent, comme auparavant, au bas de l'échelle, ainsi que les catégories "nuisances publiques avec victime», «infractions contre l'État" et "auto» (délits concernant l'usage des automobiles).

\section{LA RÉPARTITION DES PRISONNIERS OU DES CONDAMNÉS SELON LE TYPE DE SENTENCE}

Comme dans la section précédente, il faut distinguer les deux périodes de notre étude. En effet, d'une part, les documents parlementaires qui rapportent les différentes sentences encourues par les détenus (et non par tous les sujets admis) les répartissent en cinq catégories, condamnation à une peine d'amende, à l'École de réforme, à la prison, au pénitencier et à mort. D'autre part, dans les registres d'écrou des années 1931-1940, la colonne relative au type de sentence rend compte des décisions juridictionnelles en des termes plus larges que dans la précédente période, puisqu'elle concerne non seulement les détenus, mais les prévenus.

Ayant été dans l'impossibilité de former des catégories homogènes de sentences pour l'ensemble des années 1912-1940, nous devons étudier en deux temps le mouvement de la population de la prison, relativement à cette variable.

La répartition des détenus selon le type de sentence (1912-1930)

1. Une première remarque s'impose quant aux condamnations à l'amende et à l'École de réforme. Dans les deux cas, elles n'ont été prononcées que d'une manière épisodique, pendant les années 1912,1913,1914, 1915, 1918 et 1920, quant à l'amende, et pendant les années 1915 à 1917, quant à l'envoi dans une école de réforme. Ensuite, il est étonnant de constater qu'en un an, les sentences à une peine d'amende passent de 1 en 1912 à 4077 en 1913, comme on le constate sur la base du tableau statistique 8 :

On peut avancer l'hypothèse selon laquelle les amendes n'étaient plus considérées comme type de sentence - et ce, de façon constante - à partir de 1921. En effet, dans les registres d'écrou des années 1931-1940, les peines d'amende sont toujours inscrites alternativement à une peine d'emprisonnement. Il semble donc qu'à partir de 1921 surtout, il y a eu une nouvelle façon d'inscrire les peines d'amende dans les registres. C'est pourquoi les amendes, dès 1921, ne sont plus retenues dans les statistiques 
TABLEAU 8

Répartition des détenus selon le type de sentence

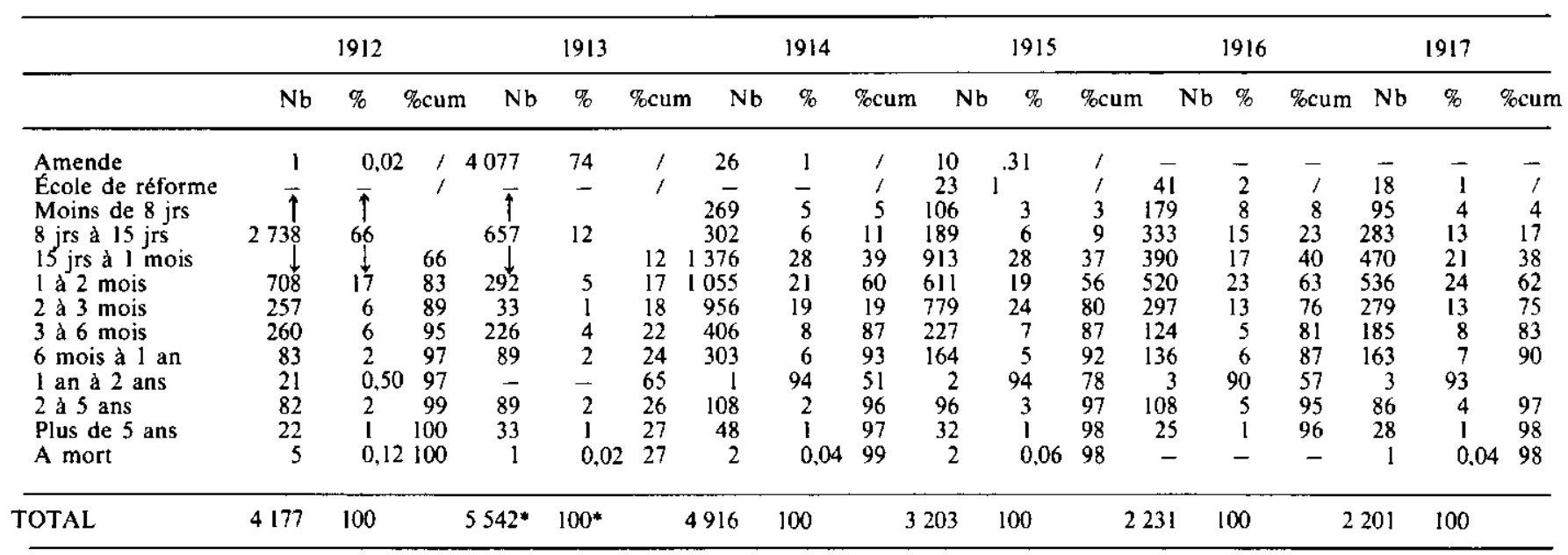

* inclus $=22$ contraintes par corps et 23 condamnés pour dettes: respectivement $.40 \%$ et $.41 \%$. 
TABLEAU 8A (suite)

Répartition des détenus selon le type de sentence

\begin{tabular}{|c|c|c|c|c|c|c|c|c|c|c|c|c|c|c|c|c|c|c|}
\hline & \multicolumn{3}{|c|}{1918} & \multicolumn{3}{|c|}{1919} & \multicolumn{3}{|c|}{1920} & \multicolumn{3}{|c|}{1921} & \multicolumn{3}{|c|}{1922} & \multicolumn{3}{|c|}{1923} \\
\hline & $\mathrm{Nb}$ & $\%$ & \%cum & $\mathrm{Nb}$ & $\%$ & \%cum & $\mathrm{Nb}$ & $\%$ & $\% \mathrm{cum}$ & $\mathrm{Nb}$ & $\%$ & \%cum & $\mathrm{Nb}$ & $\%$ & \%cum & $\mathrm{Nb}$ & $\%$ & \%cum \\
\hline Amende & 356 & 14 & 1 & - & - & - & 72 & 2 & / & - & - & - & - & - & - & - & - & - \\
\hline École de réforme & - & - & - & - & - & - & - & - & - & - & - & - & - & - & - & - & - & - \\
\hline Moins de 8 jurs & 20 & 1 & l & 63 & 2 & 2 & - & - & - & 591 & 12 & 12 & 746 & 17 & 17 & 565 & 15 & 15 \\
\hline 8 a 15 jrs & 210 & 8 & 9 & 729 & 24 & 26 & 1272 & 42 & 42 & 1460 & 30 & 42 & 372 & 8 & 25 & 338 & 9 & 24 \\
\hline 15 jrs à I mois & 699 & 27 & 36 & 368 & 12 & 38 & 845 & 28 & 70 & 1041 & 21 & 63 & 537 & 12 & 37 & 498 & 14 & 38 \\
\hline 1 à 2 mois & 494 & 19 & 55 & 741 & 25 & 63 & 265 & 9 & 79 & 913 & 19 & 82 & 912 & 21 & 58 & 969 & 26 & 64 \\
\hline 2 à 3 mois & 253 & 10 & 65 & 313 & 10 & 73 & 171 & 6 & 85 & 247 & 5 & 87 & 369 & 8 & 66 & 281 & 8 & 72 \\
\hline 3 a 6 mois & 152 & 6 & 71 & 214 & 7 & 80 & 177 & 6 & 91 & 256 & 5 & 92 & 498 & 11 & 77 & 384 & 10 & 82 \\
\hline 6 mois à 1 an & 190 & 7 & 78 & 198 & 7 & 87 & 67 & 2 & 93 & 139 & 3 & 95 & 432 & 10 & 87 & 330 & 9 & 91 \\
\hline 1 à 2 ans & 113 & 4 & 82 & 83 & 3 & 90 & 4 & 0.13 & 393 & 100 & 2 & 97 & 272 & 6 & 93 & 163 & 4 & 95 \\
\hline 2 à 5 ans & 38 & 1 & 83 & 228 & 8 & 98 & 113 & 4 & 97 & 140 & 3 & 100 & 197 & 4 & 97 & 127 & 3 & 98 \\
\hline Plus de 5 ans & 19 & $i$ & 84 & 61 & & 100 & 18 & i & 98 & 33 & 1 & 101 & 56 & 1 & 98 & 20 & 1 & 99 \\
\hline A mort & 3 & 0.08 & $88 \quad 85$ & 6 & 0.2 & $20 ! 00$ & - & - & - & - & - & & - & - & & 1 & 0.03 & 399 \\
\hline TOTAL & 2546 & 100 & & 3004 & 100 & & 3004 & 100 & & 4920 & 100 & & 4391 & 100 & & 3676 & 100 & \\
\hline
\end{tabular}


TABLEAU 8B (suite)

Répartition des détenus selon le type de sentence

\begin{tabular}{|c|c|c|c|c|c|c|c|c|c|c|c|c|c|c|c|c|c|c|c|c|c|}
\hline & \multicolumn{3}{|c|}{1924} & \multicolumn{3}{|c|}{1925} & \multicolumn{3}{|c|}{1926} & \multicolumn{3}{|c|}{1927} & \multicolumn{3}{|c|}{1928} & \multicolumn{3}{|c|}{1929} & \multicolumn{3}{|c|}{1930} \\
\hline & Nb & $\%$ & \%cum & $\mathrm{Nb}$ & $\%$ & \%cum & $\mathrm{Nb}$ & $\%$ & \%cum & $\mathrm{Nb}$ & $\% \%$ & \%cum & $\mathrm{Nb}$ & $\%$ & \%cum & $\mathrm{Nb}$ & $\%$ & \%cum & $\mathrm{m} \quad \mathrm{Nb}$ & $\%$ & $\%$ cum \\
\hline Amende & - & - & - & - & - & - & - & - & - & - & - & - & - & - & - & - & - & - & - & - & - \\
\hline École de réforme & - & - & - & - & - & - & - & - & - & - & - & - & - & - & - & - & - & - & - & - & - \\
\hline Moins de 8 jrs & 602 & 15 & 15 & 806 & 17 & 17 & 490 & 13 & 13 & 554 & 13 & 13 & 253 & 6 & 6 & 694 & 15 & 15 & 920 & 20 & 20 \\
\hline 8 à 15 jrs & 299 & 7 & 22 & 471 & 10 & 27 & 586 & 16 & 29 & 395 & 9 & 22 & 236 & 6 & 121 & 1004 & 21 & 36 & 917 & 20 & 40 \\
\hline 15 jrs à 1 mois & 658 & 16 & 38 & 693 & 15 & 42 & 443 & 12 & 41 & I 277 & 30 & 52 & 1317 & 34 & 46 & 808 & 17 & 53 & 585 & 12 & 52 \\
\hline 2 à 3 mois & 322 & 8 & 65 & 295 & 6 & 64 & 279 & 7 & 58 & 251 & 6 & 69 & 254 & 6 & 64 & 263 & 6 & 71 & 208 & 4 & 68 \\
\hline 3 aे 6 mois & 647 & 16 & 81 & 801 & 17 & 81 & 839 & 23 & 81 & 729 & 17 & 86 & 725 & 19 & 83 & 723 & 15 & 86 & 641 & 14 & 82 \\
\hline 6 mois à 1 an & 326 & 8 & 89 & 347 & 7 & 88 & 281 & 8 & 89 & 216 & 5 & 91 & 264 & 7 & 90 & 315 & 7 & 93 & 356 & 8 & 90 \\
\hline I à 2 ans & 186 & 5 & 94 & 177 & 4 & 92 & 226 & 6 & 95 & 141 & 3 & 94 & 159 & 4 & 94 & 166 & 4 & 97 & 259 & 6 & 96 \\
\hline 2 à 5 ans & 212 & & 99 & 218 & 5 & 97 & 178 & 5 & 100 & 163 & 4 & 98 & 176 & 4 & 98 & $|S|$ & 3 & 100 & 185 & 4 & 100 \\
\hline Plus de 5 ans & 41 & & 100 & 100 & 2 & 99 & 27 & l & 101 & 39 & 1 & 99 & 44 & i & 99 & 51 & 1 & 101 & 48 & 1 & 101 \\
\hline A mort & 9 & 0,2 & 22100 & 4 & 0.09 & $19 \quad 99$ & - & - & & 2 & 0.05 & 599 & 2 & 0.05 & $5 \quad 99$ & 5 & 0,1 & 0101 & 5 & & 11101 \\
\hline
\end{tabular}


officielles, de même que, de 1931 à 1940 , il n'y a aucune catégorie de sentence concernant uniquement l'amende.

2. Les condamnations à une peine de prison forment la grande majorité des sentences rendues, puisque - mis à part l'année 1913 où, on constate au contraire, une majorité d'amende (74\%) - elles dépassent le plus souvent un taux de 93\%, sauf en $1916(92 \%)$, $1918(84 \%)$ et $1919(90 \%)$.

Plus précisément, plus de la moitié des sentences concerne des peines de 1 jour à 2 mois d'emprisonnement. Elles varient entre $5 \%$ en 1926 et $82 \%$ en 1912 , en excluant encore l'année 1913 où les sentences de 1 jour à 2 mois n'atteignent que $17,12 \%$. Ces résultats correspondent à ceux obtenus par MM. Menguile et Pépin pour 1972-1973'.

Quant aux sentences d'incarcération de 1 jour à 6 mois, les tableaux indiquent toujours mis à part l'année 1913, un taux cumulé variant entre un minimum de $72 \%$ en 1918 et un maximum de $9 \%$ en 1920 .

Le taux de condamnations de 6 mois à 1 an est assez faible, avec un minimum de $2 \%$ en 1913 et $10 \%$ en 1922 .

3. Les sentences au pénitencier varient de $2 \%$ en 1913 , à $10 \%$ en 1919. Les condamnations à plus de 5 ans sont moins nombreuses que celles de 2 à 5 ans. En effet, elles s'échelonnent de $0,53 \%$ en 1912 , à $2 \%$ en 1925, tandis que les sentences à moins de 5 ans de pénitencier varient de $1 \%$ en 1918 à $8 \%$ en 1919.

Aucune condamnation à mort n'a été encourue en 1916, 1920 à 1922, et 1926. C'est en 1924 que l'on trouve un maximum de 9 peines de mort.

Les tableaux 9-9A rapportent la répartition des détenus selon le type de sentence, de 1931 à 1940. Leur analyse donne les résultats suivants, légèrement inférieurs à la réalité - précisons le bien car ces tableaux excluent la catégorie "autres» qui comprend un certain nombre de détenus, impossible à distinguer de celui des prévenus.

1. L'ordre décroissant d'importance, selon lequel les sentences sont rendues, se présente ainsi; en premier lieu, les condamnations à moins de 15 jours constituent $25 \%$ en moyenne des sentences

5. Description de la population de l'établissement de détention de Montréal, par Claude MENGHILE et Ronald PÉPIN, juin 1974. p. 17. 
TABLEAU 9

Répartition des détenus selon le type de sentence

\begin{tabular}{|c|c|c|c|c|c|c|c|c|c|c|c|c|}
\hline & $\lfloor 93\rfloor$ & & 1932 & & 1933 & & 1934 & & 1935 & & 1936 & \\
\hline & $\%$ & \%cum & $\%$ & \%cum & $\%$ & \%cum & $\%$ & \%cum & $\%$ & \%cum & $\%$ & \%cum \\
\hline $\begin{array}{l}\text { Moins de } 15 \text { jrs } \\
15 \text { jrs à } 1 \text { mois } \\
1 \text { à } 2 \text { mois } \\
2 \text { à } 3 \text { mois } \\
3 \text { à } 6 \text { mois } \\
6 \text { mois à } 1 \text { an } \\
1 \text { an à } 2 \text { ans } \\
2 \text { à } 5 \text { ans } \\
\text { Plus de } 5 \text { ans }\end{array}$ & $\begin{array}{r}31 \\
13 \\
8 \\
9 \\
11 \\
13 \\
6 \\
7 \\
2\end{array}$ & $\begin{array}{r}31 \\
44 \\
52 \\
61 \\
72 \\
85 \\
91 \\
98 \\
100\end{array}$ & $\begin{array}{r}34 \\
14 \\
9 \\
3 \\
12 \\
7 \\
11 \\
7 \\
3\end{array}$ & $\begin{array}{r}34 \\
48 \\
57 \\
60 \\
72 \\
79 \\
90 \\
97 \\
100\end{array}$ & $\begin{array}{r}22 \\
16 \\
18 \\
8 \\
7 \\
7 \\
9 \\
13 \\
1\end{array}$ & $\begin{array}{r}22 \\
38 \\
56 \\
64 \\
71 \\
78 \\
87 \\
100 \\
101\end{array}$ & $\begin{array}{r}21 \\
11 \\
20 \\
6 \\
11 \\
10 \\
7 \\
11 \\
3\end{array}$ & $\begin{array}{r}21 \\
32 \\
52 \\
58 \\
69 \\
79 \\
86 \\
97 \\
100\end{array}$ & $\begin{array}{r}21 \\
10 \\
21 \\
5 \\
16 \\
12 \\
9 \\
3 \\
3\end{array}$ & $\begin{array}{r}21 \\
31 \\
52 \\
57 \\
73 \\
85 \\
94 \\
97 \\
100\end{array}$ & $\begin{array}{r}32 \\
20 \\
8 \\
9 \\
11 \\
6 \\
10 \\
2 \\
1\end{array}$ & $\begin{array}{l}32 \\
52 \\
60 \\
69 \\
80 \\
86 \\
96 \\
98\end{array}$ \\
\hline TOTAL & 100 & 100 & 100 & 100 & 100 & 100 & 100 & 100 & 100 & 100 & 100 & 98 \\
\hline
\end{tabular}


TABLEAU 9A (SUITE)

Répartition des détenus selon le type de sentence

\begin{tabular}{|c|c|c|c|c|c|c|c|c|c|c|}
\hline & \multirow{2}{*}{$\frac{1937}{\%}$} & \multirow{2}{*}{\multicolumn{2}{|c|}{$\frac{1938}{\%}$}} & \multirow[b]{2}{*}{ \%cum } & \multirow{2}{*}{$\frac{1939}{\%}$} & \multicolumn{3}{|c|}{1940} & \multicolumn{2}{|c|}{$\begin{array}{c}\text { TOTAL } \\
1931 \text { à } 1940 \\
\end{array}$} \\
\hline & & & & & & $\%$ cum & $\%$ & \%cum & $\%$ & \%cum \\
\hline $\begin{array}{l}\text { Moins de } 15 \text { jrs } \\
15 \text { jrs à } 1 \text { mois } \\
1 \text { à } 2 \text { mois } \\
2 \text { à } 3 \text { mois } \\
3 \text { à } 6 \text { mois } \\
6 \text { mois à } 1 \text { an } \\
1 \text { à } 2 \text { ans } \\
2 \text { à } 5 \text { ans } \\
\text { Plus de } 5 \text { ans }\end{array}$ & $\begin{array}{r}25 \\
28 \\
18 \\
7 \\
9 \\
3 \\
2 \\
5 \\
3\end{array}$ & $\begin{array}{r}25 \\
53 \\
71 \\
78 \\
87 \\
90 \\
92 \\
97 \\
100\end{array}$ & $\begin{array}{r}20 \\
23 \\
14 \\
4 \\
7 \\
11 \\
12 \\
9 \\
1\end{array}$ & $\begin{array}{r}20 \\
43 \\
57 \\
61 \\
68 \\
79 \\
91 \\
100 \\
101\end{array}$ & $\begin{array}{r}26 \\
23 \\
9 \\
7 \\
14 \\
8 \\
8 \\
4 \\
-\end{array}$ & $\begin{array}{l}26 \\
49 \\
58 \\
65 \\
79 \\
87 \\
95 \\
99\end{array}$ & $\begin{array}{r}20 \\
20 \\
19 \\
5 \\
12 \\
6 \\
6 \\
10 \\
2\end{array}$ & $\begin{array}{r}20 \\
40 \\
59 \\
64 \\
76 \\
82 \\
88 \\
98 \\
100\end{array}$ & $\begin{array}{r}25 \\
18 \\
14 \\
6 \\
11 \\
8 \\
8 \\
7 \\
2\end{array}$ & $\begin{array}{l}25 \\
43 \\
57 \\
63 \\
74 \\
82 \\
90 \\
97 \\
99\end{array}$ \\
\hline TOTAL & 100 & 100 & 100 & 100 & 100 & 99 & 100 & 100 & 100 & 99 \\
\hline
\end{tabular}


prononcées (taux minimum $=20 \%$, en $1940-$ taux maximum $=34 \%$ en 1932). Au deuxième rang. se situent les sentences de 15 jours à 1 mois avec un taux moyen s'élevant à $18 \%$ et variant de $10 \%$, en 1935 à $28 \%$ en 1937. Les sentences de 1 à 2 mois viennent en troisième position avec un pourcentage moyen de $14 \%$ $($ minimum $=8 \%$ en $1931-$ maximum $=21 \%$ en 1935$)$. Puis se trouvent les sentences de 3 à 6 mois avec un taux moyen de $11 \%$ qui s'échelonne de $7 \%$ en 1938 à $16 \%$ en 1935. Les sentences de 2 à 3 mois, 6 mois à 1 an, un à 2 ans et 2 à 5 ans se situent dans un même ordre de grandeur, entre des taux moyens de $6 \%$ ( 2 à 3 mois), et $8 \%$ (6 mois à 1 an). Enfin, au bas de l'échelle, les condamnations à plus de 5 ans atteignent un taux moyen de $2 \%$, variant de $0 \%$ en 1936 et 1939, à 3\% en 1937.

2. Par ailleurs, l'examen des taux cumulés comparé à celui de la période précédente nous permet de faire les constatations suivantes.

Les sentences d'emprisonnement constituent toujours une très forte majorité, atteignant un pourcentage moyen de $91 \%$ qui varie de $86 \%$, en 1933 à $98 \%$ en 1936.

En particulier, les sentences de 1 jour à 2 mois dépassent à elles seules la moitié des sentences prononcées, comme durant la période précédente, avec un taux moyen de $57 \%$, variant de $52 \%$, en 1931 , à $71 \%$ en 1937 .

Enfin, les taux de sentence de 1 jour à 6 mois peuvent être rapprochés également de ceux rapportés quant aux années 19121930. Le taux cumulé moyen est de $75 \%$ avec un minimum de $68 \%$ en 1938 et un maximum de $87 \%$, en 1937.

La répartition des prisonniers d'après l'amende est indiquée au tableau 10.

En premier lieu, le taux des sujets incarcérés qui n'ont été condamnés à aucune peine d'amende atteint une moyenne de $52 \%$, entre un minimum de $46 \%$ en 1937 et un maximum de $65 \%$ en 1933.

Quant au pourcentage moyen des prisonniers qui ont encouru une peine d'amende, il est de $47 \%$, mais il convient de rappeler qu'il concerne une telle sentence prononcée toujours alternativement à une peine d'emprisonnement. 
TABLEAU 10

Répartition des prisonniers d'après l'amende

\begin{tabular}{|c|c|c|c|c|c|c|c|}
\hline & Pas d'amende & & Amende payée & & $\begin{array}{c}\text { Amende } \\
\text { non payée }\end{array}$ & & Total \\
\hline & $\%$ & & $\%$ & & $\%$ & & $\%$ \\
\hline $\begin{array}{l}1931 \\
1932 \\
1933 \\
1934 \\
1935 \\
1936 \\
1937 \\
1938 \\
1939 \\
1940\end{array}$ & $\begin{array}{l}52,4 \\
49,5 \\
64,7 \\
53,3 \\
52,2 \\
49,7 \\
46,0 \\
58,3 \\
46,8 \\
50,6\end{array}$ & & $\begin{array}{l}19,3 \\
17,2 \\
15,8 \\
22,8 \\
27,8 \\
25,5 \\
21,4 \\
16,1 \\
22,0 \\
15.7\end{array}$ & & $\begin{array}{r}28,3 \\
33,3 \\
19,6 \\
23,9 \\
20,0 \\
24,8 \\
32,6 \\
25,5 \\
31,2 \\
33,7\end{array}$ & & $\begin{array}{r}100,0 \\
100,0 \\
100,1 \\
100,0 \\
100,0 \\
100,0 \\
100,0 \\
99,9 \\
100,0 \\
100,0\end{array}$ \\
\hline $\begin{array}{l}\text { Moyenne } \\
1931-1940\end{array}$ & 52,4 & 375 & 20,2 & 507 & 27.3 & 1854 & 99,9 \\
\hline
\end{tabular}

En moyenne $27 \%$ des détenus n'ont pas payé leur amende (taux le plus bas $=20 \%$, en 1933 - le taux le plus élevé $=34 \%$, en 1940), tandis que $20 \%$ en moyenne des détenus l'ont payée (taux inférieur $=16 \%$ en $1940-$ taux supérieur $=28 \%$ en 1935).

\section{E. LA RÉPARTITION DES ADMISSIONS D'APRÈS L'ÂGE}

\section{La période 1914-1930}

Les tableaux 11-11A concernant la répartition des prisonniers d'après leur âge indiquent les résultats suivants.

Le taux des individus âgés de 30 à 39 ans dépasse celui des autres classes d'âge. En effet, il varie entre un niveau inférieur de $24 \%$, en 1919 et un niveau supérieur de $30 \%$ en 1925 , tandis que les autres groupes, spécialement ceux âgés de moins de 50 ans, représentent des pourcentages moins élevés et, en outre, assez comparables. C'est ainsi que :

a) le taux des sujets âgés de 16 à 19 ans s'élève d'un minimum de $7 \%$ en 1916 , à un maximum de $13 \%$ en 1919 ;

b) le taux du groupe 20-24 ans atteint un plancher de $14 \%$ en 1925 , et un plafond de $18 \%$ en 1920 ;

c) la classe 25-29 ans s'échelonne entre les taux $13 \%$ en 1916 et $20 \%$ en 1928 ; 
TABLEAU 11

Répartition des prisonniers d'après leur âge

\begin{tabular}{|c|c|c|c|c|c|c|c|c|c|c|c|c|c|c|c|c|c|c|c|c|c|}
\hline & \multicolumn{3}{|c|}{1914} & \multicolumn{3}{|c|}{1915} & \multicolumn{3}{|c|}{1916} & \multicolumn{3}{|c|}{1917} & \multicolumn{3}{|c|}{1918} & \multicolumn{3}{|c|}{1919} & \multicolumn{3}{|c|}{1920} \\
\hline & $\mathrm{Nb}$ & $\%$ & \%cum & $\mathrm{Nb}$ & $\%$ & \%cum & $\mathrm{Nb}$ & $\%$ & \%cum & $\mathrm{Nb}$ & $\%$ & $\%$ cum & $\mathrm{Nb}$ & $\%$ & $\%$ cum & $\mathrm{Nb}$ & $\%$ & \%cum & $\mathrm{Nb}$ & $\%$ & \%cum \\
\hline 14.15 ans & - & - & - & - & - & - & - & - & - & - & - & - & 87 & 2 & 2 & - & - & - & - & - & - \\
\hline 16.19 ans & 764 & 11 & 11 & 428 & 9 & 9 & 254 & 7 & 7 & 390 & 11 & 11 & $40 \mathrm{I}$ & 10 & 12 & 568 & 13 & 13 & 456 & 10 & 10 \\
\hline $20-24$ ans & 1093 & 16 & 27 & 747 & 17 & 26 & 489 & 14 & 21 & 553 & 15 & 26 & 567 & 14 & 26 & 697 & 16 & 29 & 790 & 18 & 28 \\
\hline 25.29 ans & 1067 & 16 & 43 & 704 & 16 & 42 & 455 & 13 & 34 & 509 & 14 & 40 & 537 & 14 & 40 & 580 & 13 & 42 & 659 & 15 & 43 \\
\hline 30.39 ans & 1696 & 25 & 68 & 1117 & 25 & 67 & 880 & 26 & 60 & 913 & 25 & 65 & 1023 & 26 & 66 & 1052 & 24 & 66 & 1196 & 27 & 70 \\
\hline 40.49 ans & 1105 & 17 & 85 & 758 & 17 & 84 & 650 & 19 & 79 & 674 & 19 & 84 & 785 & 20 & 86 & 792 & 18 & 84 & 730 & 17 & 87 \\
\hline $50-59$ ans & 672 & 10 & 95 & 501 & 11 & 95 & 408 & 12 & 91 & 361 & 10 & 94 & 365 & 9 & 95 & 409 & 9 & 93 & 401 & 9 & 96 \\
\hline 60 ans et + & 280 & 4 & 99 & 261 & 6 & 101 & 262 & 8 & 99 & 215 & 6 & 100 & 191 & 5 & 100 & 223 & 5 & 98 & 175 & 4 & 100 \\
\hline TOTAL & 6677 & & & 4516 & & & 3398 & & & 3615 & & & 3956 & & & 4321 & & & 4407 & & \\
\hline
\end{tabular}


TABLEAU IIA (SUITE)

Répartition des prisonniers d'après leur âge

\begin{tabular}{|c|c|c|c|c|c|c|c|c|c|c|c|c|c|c|c|c|c|c|c|c|c|c|c|c|c|c|c|c|c|c|}
\hline \multirow[b]{3}{*}{$14-15$ ans } & \multicolumn{3}{|c|}{1921} & \multicolumn{3}{|c|}{1922} & \multicolumn{3}{|c|}{1923} & \multicolumn{3}{|c|}{1924} & \multicolumn{3}{|c|}{1925} & \multicolumn{3}{|c|}{1926} & \multicolumn{3}{|c|}{1927} & \multicolumn{3}{|c|}{1928} & \multicolumn{3}{|c|}{1929} & \multicolumn{3}{|c|}{1930} \\
\hline & \multirow{2}{*}{$\begin{array}{c}\mathrm{Nb} \\
-\end{array}$} & \multicolumn{2}{|c|}{$\%$ \%cum } & \multirow{2}{*}{$\begin{array}{r}\text { NB } \\
-\end{array}$} & \multicolumn{2}{|c|}{ \% \%cum } & \multirow{2}{*}{$\begin{array}{c}\mathrm{Nb} \\
-\end{array}$} & \multicolumn{2}{|c|}{$\%$ \%cum } & \multirow{2}{*}{$\mathrm{Nb}_{-}^{\mathrm{Nb}}$} & \multicolumn{2}{|c|}{$\%$ coum } & \multirow{2}{*}{$\underbrace{\mathrm{Nb}}_{-}$} & \multicolumn{2}{|c|}{ rocum } & \multirow{2}{*}{$\begin{array}{r}\mathrm{Nb} \\
-\end{array}$} & \multicolumn{2}{|c|}{ * *cum } & \multirow{2}{*}{$\begin{array}{c}\mathrm{Nb} \\
-\end{array}$} & \multicolumn{2}{|c|}{$\%$ teum } & \multirow{2}{*}{$\begin{array}{c}\mathrm{Nb} \\
?\end{array}$} & \multicolumn{2}{|c|}{8 scum } & \multirow{2}{*}{$\frac{\mathrm{Nb}}{6}$} & \% & coun & $\mathrm{Nb}$ & & \%cum \\
\hline & & - & - & & - & - & & - & - & & - & - & & - & - & & - & - & & - & - & & .03 & .03 & & .08 & .08 & 1 & .01 & .01 \\
\hline $16-19$ ans & 634 & 10 & 10 & 477 & 9 & 9 & 403 & 8 & 8 & 582 & 10 & 10 & 533 & 8 & 8 & 560 & 10 & 10 & 613 & 10 & 10 & 577 & 9 & 9 & 716 & 10 & 10 & 845 & 12 & 12 \\
\hline $20-24$ ans & 1087 & 16 & 26 & 906 & 16 & 25 & 772 & 15 & 23 & 896 & 15 & 25 & 925 & 14 & 22 & 973 & 17 & 27 & 971 & 15 & 25 & 891 & 15 & 24 & 1049 & 14 & 24 & 1190 & 17 & 29 \\
\hline 25.29 ans & 986 & 15 & 41 & 878 & 16 & 41 & 990 & 19 & 42 & 1134 & 19 & 44 & 1142 & 17 & 39 & 1011 & 18 & 45 & 1119 & 18 & 43 & 1241 & 20 & 44 & 1372 & 19 & 4.3 & 1349 & 19 & 48 \\
\hline $40-49$ ans & 1238 & 19 & 85 & 973 & 18 & 85 & 889 & 17 & 86 & 1004 & 17 & 88 & 1104 & 17 & 86 & 894 & 16 & 87 & 1074 & 17 & 86 & 972 & 16 & 86 & 1143 & 16 & 45 & 984 & 14 & 89 \\
\hline 50.59 ans & 727 & 11 & 96 & 535 & 10 & 95 & 505 & 10 & 96 & 498 & 8 & 96 & 606 & 9 & 95 & 478 & 8 & 95 & 632 & 10 & 96 & 542 & $y$ & 95 & 718 & 10 & 95 & 494 & 7 & 96 \\
\hline 60 ans et + & 309 & 5 & 101 & 299 & 5 & 100 & 264 & 5 & 101 & 274 & 5 & $10 \mathrm{t}$ & 337 & 5 & 100 & 277 & 5 & 100 & 336 & 5 & 101 & 285 & 5 & 100 & 352 & 5 & 100 & 255 & 4 & 100 \\
\hline TOTAL & 6618 & & & 5510 & & & 5234 & & & 6026 & & & 6612 & & & 5677 & & & 6373 & & & 6089 & & & 7258 & & & 6990 & & \\
\hline
\end{tabular}


d) les sujets âgés de 40 à 49 ans représentent un taux variant entre un minimum de $14 \%$ en 1930 et un maximum de $20 \%$ en 1918.

Enfin, les deux groupes âgés de plus de 50 ans atteignent les taux les plus bas. D'autre part, les sujets âgés de 50 à 59 ans constituent un pourcentage inférieur de $7 \%$ en 1930 , pour s'élever jusqu'à $12 \%$ en 1916 et, d'autre part, le groupe âgé de 60 ans et plus varie entre $4 \%$ en 1930 , et $8 \%$ en 1916 .

Quant à l'examen des pourcentages cumulés, nous constatons que :

a) les sujets âgés de moins de 25 ans atteignent un taux s'échelonnant entre $22 \%$ en 1916 , et $29 \%$ en 1919 ;

b) les personnes âgées de moins de 30 ans représentent un pourcentage variant de $35 \%$ en 1916 , à $48 \%$ en 1930 ;

c) le pourcentage des infracteurs de moins de 40 ans s'établit entre un minimum de $61 \%$ en 1916 , et un maximum de $75 \%$ en 1930 ;

d) enfin, les individus âgés de moins de 50 ans atteignent un taux passant de $80 \%$ en 1916 , à $89 \%$ en 1930 .

\section{La période 1931-1940}

Les tableaux 12-12A indiquent la répartition des prisonniers selon leur âge, de 1931 à 1940.

D'emblée, le tableau montre que la classe des sujets âgés de 30 à 39 ans - comme durant la période précédente, mais avec un taux un peu moins élevé - se trouve en première position, avec un taux de $23 \%$. Plus précisément, elle varie entre un minimum de $19 \%$ en 1932, et un maximum de $30 \%$ en 1935.

La répartition en pourcentage des autres groupes est également comparable à celle de la période 1914-1930. Elle se présente ainsi :

a) les jeunes de moins de 20 ans représentent un taux moyen - pour l'ensemble des années 1931-40 - de 14\%, qui varie entre $11 \%$, en 1937 et $16 \%$ en 1939 ;

b) le pourcentage du groupe suivant (20-24 ans) atteint une moyenne de $18 \%$ (minimum : $12 \%$ en 1939 - maximum : $23 \%$ en 1934); 
TABLEAU 12

Répartition des prisonniers d'après leur âge

\begin{tabular}{|c|c|c|c|c|c|c|c|c|c|c|c|c|}
\hline & 1931 & & 1932 & & 1933 & & 1934 & & 193 & & 1936 & \\
\hline & $\%$ & $\%$ cum & $\%$ & $\%$ cum & $\%$ & \%cum & $\%$ & $\%$ cum & $\%$ & \%cum & $\%$ & $\%$ cum \\
\hline $\begin{array}{l}15-17 \text { ans } \\
18-19 \text { ans } \\
20-24 \text { ans } \\
25-29 \text { ans } \\
30-39 \text { ans } \\
40-49 \text { ans } \\
50-59 \text { ans } \\
60 \text { ans et }+ \\
\text { Pas de renseignements }\end{array}$ & $\begin{array}{r}7 \\
9 \\
15 \\
20 \\
25 \\
16 \\
7 \\
1 \\
-\end{array}$ & $\begin{array}{r}7 \\
16 \\
31 \\
51 \\
76 \\
92 \\
99 \\
100 \\
-\end{array}$ & $\begin{array}{r}6 \\
9 \\
23 \\
16 \\
20 \\
21 \\
4 \\
2 \\
-\end{array}$ & $\begin{array}{r}6 \\
15 \\
38 \\
54 \\
74 \\
95 \\
99 \\
101\end{array}$ & $\begin{array}{r}4 \\
11 \\
16 \\
16 \\
25 \\
11 \\
14 \\
3 \\
-\end{array}$ & $\begin{array}{r}4 \\
15 \\
31 \\
47 \\
72 \\
83 \\
97 \\
100 \\
-\end{array}$ & $\begin{array}{r}6 \\
8 \\
23 \\
16 \\
21 \\
15 \\
7 \\
4 \\
-\end{array}$ & $\begin{array}{r}6 \\
14 \\
37 \\
53 \\
74 \\
89 \\
96 \\
100 \\
-\end{array}$ & $\begin{array}{r}4 \\
8 \\
16 \\
18 \\
30 \\
16 \\
7 \\
1 \\
-\end{array}$ & $\begin{array}{r}4 \\
12 \\
28 \\
46 \\
76 \\
92 \\
99 \\
100\end{array}$ & $\begin{array}{r}4 \\
9 \\
22 \\
19 \\
28 \\
12 \\
5 \\
2 \\
-\end{array}$ & $\begin{array}{r}4 \\
13 \\
35 \\
54 \\
82 \\
94 \\
99 \\
101\end{array}$ \\
\hline TOTAL & 100 & & 100 & & 100 & & 100 & & 100 & & 100 & \\
\hline
\end{tabular}


TABLEAU 12A

Répartition des prisonniers d'après leur âge

\begin{tabular}{|c|c|c|c|c|c|c|c|c|c|c|}
\hline & 1937 & & 1938 & & 1939 & & 1940 & & $\begin{array}{c}\text { TOTAL } \\
193] \text { à } 1940\end{array}$ & \\
\hline & $\%$ & \%cum & $\%$ & $\%$ cum & $\%$ & \%cum & $\%$ & \%cum & $\%$ & \%cum \\
\hline $\begin{array}{l}15-17 \text { ans } \\
18-19 \text { ans } \\
20-24 \text { ans } \\
25-29 \text { ans } \\
30-39 \text { ans } \\
40-49 \text { ans } \\
50-59 \text { ans } \\
60 \text { ans et }+ \\
\text { Pas de renseignements }\end{array}$ & $\begin{array}{r}6 \\
5 \\
16 \\
16 \\
23 \\
16 \\
8 \\
5 \\
5\end{array}$ & $\begin{array}{r}6 \\
11 \\
27 \\
43 \\
66 \\
82 \\
90 \\
95 \\
100\end{array}$ & $\begin{array}{r}5 \\
9 \\
19 \\
13 \\
20 \\
13 \\
8 \\
8 \\
4\end{array}$ & $\begin{array}{r}5 \\
14 \\
33 \\
46 \\
66 \\
79 \\
87 \\
95 \\
99\end{array}$ & $\begin{array}{r}5 \\
11 \\
12 \\
12 \\
24 \\
17 \\
7 \\
6 \\
6\end{array}$ & $\begin{array}{r}5 \\
16 \\
28 \\
40 \\
64 \\
81 \\
88 \\
94 \\
100\end{array}$ & $\begin{array}{r}5 \\
10 \\
16 \\
8 \\
20 \\
22 \\
8 \\
6 \\
5\end{array}$ & $\begin{array}{r}5 \\
15 \\
31 \\
39 \\
59 \\
81 \\
89 \\
95 \\
100\end{array}$ & $\begin{array}{r}5 \\
9 \\
18 \\
15 \\
23 \\
16 \\
8 \\
4 \\
2\end{array}$ & $\begin{array}{r}5 \\
14 \\
32 \\
47 \\
70 \\
86 \\
94 \\
98 \\
100\end{array}$ \\
\hline TOTAL & 100 & & 100 & & 100 & & 100 & & 100 & \\
\hline
\end{tabular}


c) les sujets de 25 à 29 ans s'établissent à un taux moyen de $15 \%$ (taux le plus bas : $8 \%$ en 1940 - taux le plus haut : $20 \%$ en 1931);

d) $16 \%$ de l'échantillon pour les années 1931-1940, sont âgés de 40 à 49 ans (minimum : $11 \%$ en 1933 - maximum : $22 \%$ en 1940);

e) les personnes âgées de 50 à 59 ans atteignent un pourcentage de $8 \%$ pour l'ensemble des années considérées, avec une variation de $4 \%$ en 1932 , à $14 \%$ en 1933 ;

f) en dernier lieu, la classe la moins importante quantitativement, est celle de plus de 60 ans dont le taux moyen est de $4 \%$ (minimum : $9 \%$ en 1931 - maximum : $8 \%$ en 1938).

L'étude des taux cumulés indique les résultats suivants :

a) $32 \%$ de l'échantillon représentent les jeunes de moins de 25 ans. Le taux varie de $27 \%$ en 1937 à $37 \%$ en 1932;

b) le groupe âgé de moins de 30 ans atteint un taux moyen de $47 \%$ (minimum : $38 \%$ en 1940 - maximum : $53 \%$ en 1932 );

c) les auteurs d'infractions dont l'âge est inférieur à 40 ans, constituent un taux moyen de $71 \%$ (niveau le plus bas $=57.8 \%$ en 1940 - niveau le plus élevé : $81 \%$ en 1936):

d) enfin, $87 \%$ concernent les sujets âgés de moins de 50 ans (minimum : $80 \%$ en 1938 - maximum : $94 \%$ en 1932).

\section{F. LA RÉPARTITION DES ADMISSIONS D'APRĖS LE NIVEAU D'INSTRUCTION DE 1914 à 1930}

La lecture des tableaux 13-13A implique une remarque. En effet, dans les documents de la Session, une des colonnes relatives à l'instruction est intitulée "possédant une instruction plus développéen, alors qu'elle est écrite en anglais with a little education.

Du fait que l'auteur de ces tableaux a voulu répartir les niveaux d'instruction entre ceux qui ne savent ni lire, ni écrire, d'une part, et ceux qui savent lire et écrire, d'autre part, il est à se demander s'il voulait en troisième lieu, retenir ceux qui possèdent une instruction plus - ou peu - développée. Dans chaque tableau, l'on trouve notée, sauf pour l'année 1914, l'indication "possédant une instruction plus développée» (toujours à côté de 
TABLEAU 13

Répartition des prisonniers selon linstruction

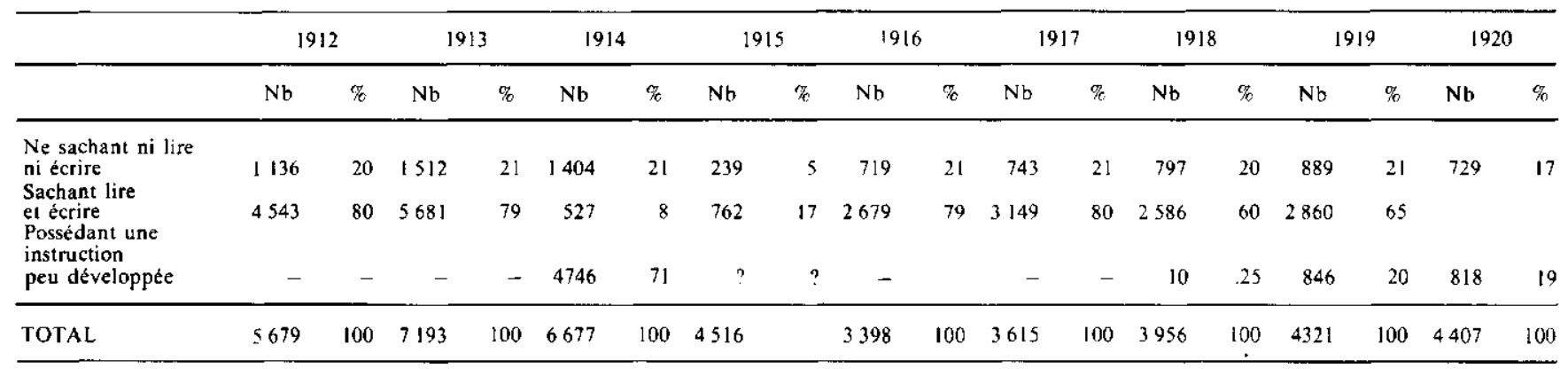


TABLEAU 13A (suite)

Répartition des prisonniers selon linstruction

\begin{tabular}{|c|c|c|c|c|c|c|c|c|c|c|c|c|c|c|c|c|c|c|c|c|}
\hline & \multicolumn{2}{|c|}{1921} & \multicolumn{2}{|c|}{1922} & \multicolumn{2}{|c|}{1923} & \multicolumn{2}{|c|}{1924} & \multicolumn{2}{|c|}{1925} & \multicolumn{2}{|c|}{1926} & \multicolumn{2}{|c|}{1927} & \multicolumn{2}{|c|}{1928} & \multicolumn{2}{|c|}{1929} & \multicolumn{2}{|c|}{1930} \\
\hline & $\mathrm{Nb}$ & $\%$ & $\mathrm{Nb}$ & $\%$ & $\mathrm{Nb}$ & $\%$ & $\mathrm{Nb}$ & $\%$ & $\mathrm{Nb}$ & $\%$ & $\mathrm{Nb}$ & $\%$ & $\mathrm{Nb}$ & $\%$ & $\mathrm{Nb}$ & $\%$ & $\mathrm{Nb}$ & $\%$ & $\mathrm{Nb}$ & 笑 \\
\hline \multirow{3}{*}{$\begin{array}{l}\text { Ne sachant } \\
\text { ni lire } \\
\text { ni écrire } \\
\text { Sachant lire } \\
\text { et écrire } \\
\text { Possédant une instruction } \\
\text { peu développée }\end{array}$} & 1051 & 16 & 938 & 17 & 682 & 13 & 655 & 11 & 892 & 13 & 387 & 7 & 1109 & 17 & I 443 & 24 & 1106 & 15 & 861 & 12 \\
\hline & 5567 & 84 & 4474 & 81 & 4443 & 85 & $5: 44$ & 85 & 5337 & 81 & 5011 & 88 & 4981 & 78 & 4646 & 76 & 5861 & 81 & 6095 & 87 \\
\hline & - & - & 98 & 2 & 109 & 2 & 227 & 4 & 383 & 6 & 279 & 5 & 283 & 4 & - & - & 291 & 4 & 34 & 49 \\
\hline TOTAL & 6618 & 100 & 5510 & 100 & 5234 & 100 & 6026 & 100 & 6612 & 100 & 5677 & 100 & 6373 & 99 & 6089 & 100 & 7258 & 100 & 6990 & 100 \\
\hline
\end{tabular}


with a little education). En 1914, la colonne indique, au contraire - et, semble-t-il, avec plus de justesse - «possédant une instruction peu développée». En 1915, cette colonne ne donne aucun chiffre. Nous avons préféré retenir comme troisième niveau d'instruction l'indication "possédant une instruction peu développée».

Il résulte de l'étude des tableaux 13-13A les constatations suivantes :

1. le taux des prisonniers sachant lire et écrire varie entre un minimum de $8 \%$ en 1914 , et un maximum de $88 \%$ en 1926. Mises à part les années $1914(8 \%), 1915(17 \%), 1919(60 \%)$ et 1920 $(65 \%)$, les sujets admis à la prison atteignent un taux toujours supérieur à $76 \%$.

2. les illettrés ou analphabètes constituent des taux variant entre un minimum de $5 \%$ en 1915 et un maximum de $24 \%$ en 1928. Ils se trouvent, en général, à un niveau supérieur à $10 \%$, sauf en $1915(5 \%)$ et en $1926(7 \%)$.

3. quant à ceux qui possèdent une instruction peu développée, il n'y en a aucun rapporté en 1912, 1913, 1916, 1917, 1921 et 1928, ni aussi en 1915 (à la suite, cette année-là, d'une erreur de transcription). D'autre part, il y en a un nombre infime par rapport au total des prisonniers : 10 en 1918 et 34, en 1930. En dehors de ces années précitées et de l'année 1914 qui voit subitement apparaître une majorité de $71 \%$ de prisonniers possédant une instruction peu développée, le pourcentage de cette catégorie varie entre un minimum de $2 \%$ en 1922 et un maximum de $20 \%$ en 1919.

L'examen de ce troisième groupe, en nous révélant des pourcentages qui varient en dents de scie, nous permet de croire à la mauvaise qualité d'enregistrement des données statistiques, des tableaux des documents de la Session.

\section{G. LA RÉPARTITION DES ADMISSIONS D'APRES LA PROFESSION, DE 1914 À 1930}

À partir des tableaux 14-14A, l'échelle des professions, par ordre décroissant, se présente ainsi :

Parmi les douze catégories de professions retenues, c'est celle des mancuvres journaliers qui vient en tête avec des pourcentages variant entre 29\% (minimum), en 1914 et 66\% (maximum), en 1929. 
TABLEAU 14

Répartition des prisonniers suivant la profession

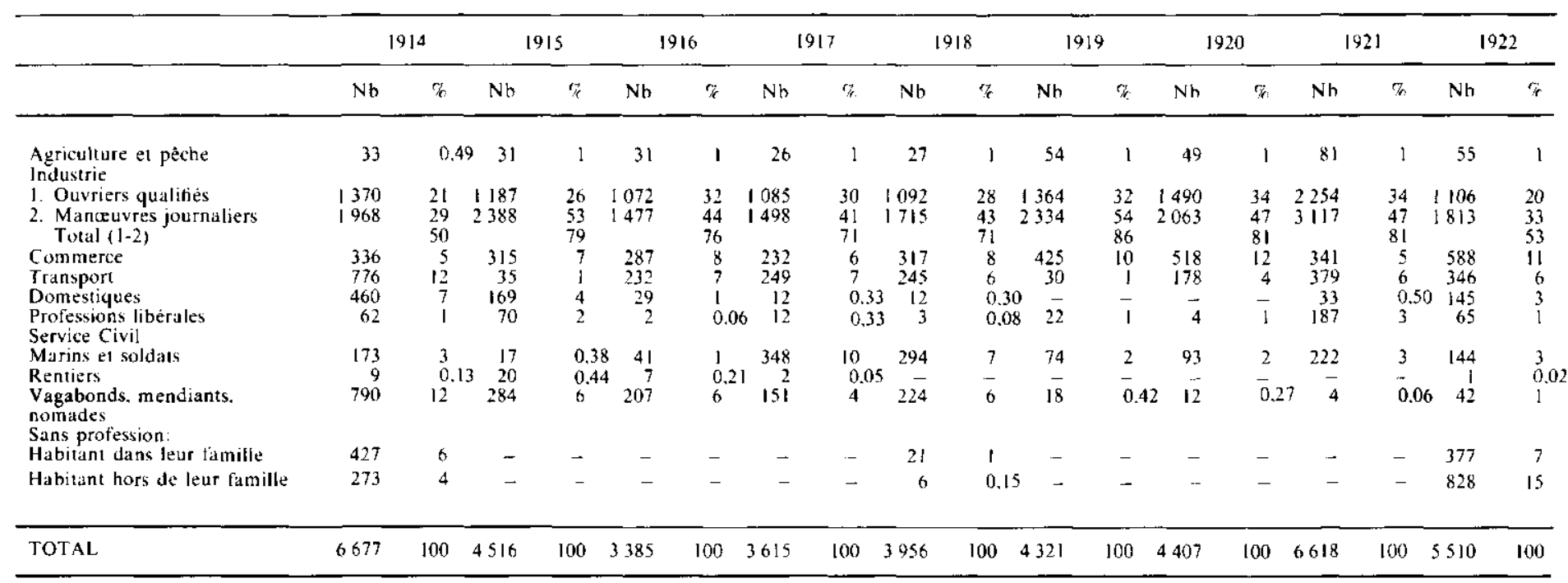


TABLEAU I4A

Répartition des prisonniers suivant la profession

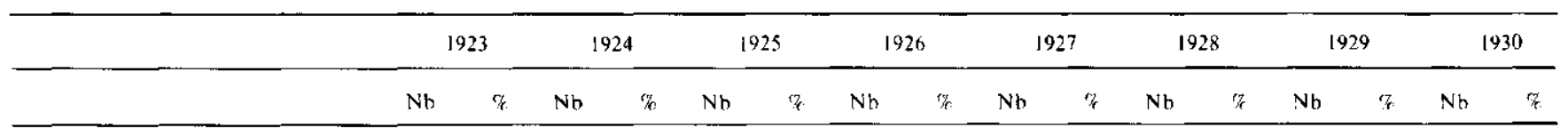

Agriculture et péche industrie:

1. Ouvriers qualifiés

2. Manceuvres journaliers

Total (1-2)

commerce

Transport

Domestiques

Prolessions libérales

service civil

Marins et soldats

Rentiers

Vagabonds, mendiants.

nomades

Sans profession:

Habitani dans leur famille

Habitant hors de leur famille

$\begin{array}{rrr}48 & 1 & 39 \\ 1851 & 35 & 2134 \\ 2523 & 48 & 3025 \\ & 83 & \\ 531 & 10 & 563 \\ 131 & 2 & 4 \\ 85 & 2 & 10 \\ 27 & 1 & 34\end{array}$

$1 \cdot 54 \quad 1 \quad 53$

-

\begin{tabular}{|c|c|c|c|c|c|c|c|c|c|c|c|c|c|c|c|c|}
\hline TOTAL & 5234 & 100 & 6026 & 100 & 6612 & 100 & 5677 & 100 & 6373 & 100 & 60189 & 100 & 7258 & 100 & 6990 & 100 \\
\hline
\end{tabular}


Suit la catégorie des ouvriers qualifiés qui, jointe à la précédente, forme le groupe dénommé "Industrie". Les taux des ouvriers qualifiés varient entre un minimum de $20 \%$, en 1922 et un maximum de $35 \%$ en 1924. Le groupe des professions «industrielles» compose donc la grande majorité des prisonniers, avec un taux s'échelonnant de $50 \%$ en 1914 à $92 \%$ en 1929 . Et, même en dehors des années $1914(50 \%)$ et $1922(53 \%)$, les prisonniers ayant eu un emploi dans le secteur de l'industrie dépassent toujours un taux de $70 \%$.

Les prisonniers provenant du secteur commercial atteignent un niveau légèrement supérieur à ceux du secteur du transport, avec des taux variant entre un minimum de 4\%, en 1929 (contre $0,03 \%$ minimum en 1925 , pour la catégorie «transport») et un maximum de $12 \%$ en 1920 (contre $12 \%$ maximum, en 1914 pour le secteur "transport»).

Les autres classes de profession (domestiques, professions libérales - service civil, marins et soldats, rentiers, vagabonds-mendiants-nomades, les sans profession, habitant soit hors, soit dans leur famille) se trouvent au bas de l'échelle avec des taux généralement inférieurs à $25 \%$.

\section{CONCLUSIONS}

Compte tenu de la documentation disponible, nous avons divisé notre analyse en deux périodes distinctes soit 1912 à 1930 et 1931 à 1940. Pour certaines analyses, nous avons pu utiliser des tableaux statistiques portant sur ces deux périodes, pour d'autres, les séries sont plus courtes. Il n'en reste pas moins que les résultats obtenus ont permis de dégager un certain nombre de constantes significatives.

On est surtout frappé par la ressemblance entre cette population et celle de la prison d'Orsainville en 1972-1973 (voir l'article de A. Campeau dans ce numéro). La très grande majorité des peines sont très courtes : de 1912 à 1940, 50 à $80 \%$ des admissions se rapportent à des peines de moins de deux mois. Même si Bordeaux reçoit tous les détenus condamnés à deux ans et moins, plus de $50 \%$ des personnes condamnées admises à Bordeaux ont de très courtes peines (moins de deux mois).

D'autre part, les délits reliés à ces condamnations sont des délits bénins qui ne sont ni des infractions contre les biens ni des infractions contre les personnes. Une très forte proportion d'entre 
eux (30 à $60 \%$ ) peuvent être classés dans la catégorie des infractions de «nuisance publique» et 30 à $50 \%$ concernent des infractions relatives à l'état d'ivresse.

De plus, même si les jeunes de moins de 30 ans sont en plus grand nombre, il est fort surprenant et significatif de remarquer que le pourcentage des délinquants âgés est fort important pour une population carcérale ou «délinquante». De 15 à $20 \%$ des admissions correspondent à des personnes âgées de 50 ans et plus de 40 ans représentent toujours environ le tiers de la population. Naturellement, cette population est très peu instruite et constituée en majorité de manœuvres ou de journaliers.

Ces données, qui, comme nous venons de le souligner, sont très étonnamment semblables à celles recueillies à Bordeaux en 1972-1973 ainsi qu'à Orsainville en 1974, démontrent clairement que les prisons provinciales du Québec ont surtout servi à enfermer les défavorisés socio-économiques. Notons aussi, et cela est très important, que l'on retrouve dans ces trois populations environ $50 \%$ des admissions pour non-paiement d'amende.

Évidemment, tous ces individus avaient commis une infraction, ou étaient dans un état que l'on qualifie d'infraction (exemple : état d'ivresse sur la voie publique). Mais il est tout aussi évident qu'ils ont été incarcérés avant tout à cause de leur situation de marginalité de défavorisés socio-économiques. Nos prisons ont servi et servent encore à nous débarrasser d'un certain nombre d'individus qui nous gênent (l'article de $\mathrm{M}^{*}$ Jean Hétu dans ce numéro, jette une lumière particulière sur le mécanisme qui produit une telle injustice).

Notre recherche, l'une des premières de ce genre au Québec, tente de présenter une image aussi réelle que possible, compte tenu des statistiques disponibles, d'une des plus importantes prisons provinciales. De telles études, comme celle de Ghislaine Julien publiée dans ce numéro, contribueront, espérons-nous, à accumuler des données permettant de mieux comprendre ce qu'a été chez nous la réaction pénale face aux marginaux, c'est-à-dire à ceux qu'on a voulu écarter de la société. 